\begin{tabular}{|l|}
\hline Access this article online \\
\hline Quick Response Code: \\
\hline Website: \\
Www.jponline.org \\
\hline DOI: \\
10.4103/JLP.JLP_46_19 \\
\hline
\end{tabular}

Departments of Microbiology and ${ }^{1}$ Pediatrics, JSS Medical College and Hospital, JSSAHER, Mysore,

Karnataka, India

Address for correspondence: Dr. Vidyavathii B.

Chittaragi,

Department of

Microbiology, JSS Medical College and Hospital, JSSAHER, Mysore, Karnataka, India. E-mail: vidyavinayakik@ gmail.com

Submission: $30-04-2019$ Accepted: 26-08-2019

\title{
A fatal, rare case of bilateral, upper, lower limbs and abdominal gangrene associated with brucellosis
}

\author{
Tejashree Anantha, Vidyavathii B. Chittaragi, M. Krishna Karthik, \\ Jagadeesh Kumar', B. Sukesh Kumar, Badveti Satya Sai
}

\section{Abstract:}

Brucellosis distributed worldwide is a classical zoonotic disease. Complications associated with gangrene are quite uncommon and may or might not occur during the course of the disease. Here, we report a pediatric age group patient who presented with fever and gangrene over the upper and lower limbs and abdomen associated with brucellosis.

Key words:

Brucella melitensis, brucellosis, early laboratory diagnosis, zoonotic disease

\section{Introduction}

$\mathrm{B}^{\mathrm{r}}$ rucellosis is one of the most commonly encountered zoonotic diseases predominantly caused by Brucella melitensis, Brucella abortus, and Brucella suis, with an estimated incidence of 5 lakh new cases/year. ${ }^{[1]}$ Infections in humans may be due to the direct contact with infected animals, inhalation of feces, or may be consumption of infected animal products. Human-to-human transmission is rare and occurs through blood transfusion, transplantation procedures, especially bone marrow transplantation, sexual mode, laboratory procedures, perinatal transmission, or through breast milk feeding. ${ }^{[2]}$ Clinical manifestations of typical acute brucellosis include usually nonspecific and patients usually present with fever, asthenia, weight loss, and back pain and the most common physical examination would be hepatosplenomegaly; cardiovascular involvement is rare, and endocarditis is the most common involvement. ${ }^{[3-5]}$

This is an open access journal, and articles are distributed under the terms of the Creative Commons Attribution-NonCommercial-ShareAlike 4.0 License, which allows others to remix, tweak, and build upon the work non-commercially, as long as appropriate credit is given and the new creations are licensed under the identical terms.

For reprints contact: reprints@medknow.com
Difficulty in diagnosis may be due to the varying clinical manifestations and underreporting can be the major cause of underestimation of the incidence. ${ }^{[6,7]}$ Most of the industrialized countries have already achieved veterinarian control of brucellosis successfully with vaccination policies, but the disease still poses a serious threat to the human population living in developing countries and in people traveling to the regions where the disease is endemic. ${ }^{[8,9]}$ Low infective doses and high rate of infectivity make this pathogen a potential biological warfare. ${ }^{[10]}$ Therefore, early laboratory diagnosis of brucellosis has a paramount importance for proper and adequate case management. Here, we report a fatal case of bilateral upper and lower limbs and abdominal gangrene associated with brucellosis in a pediatric patient.

\section{Case Report}

A 5-year-old male child was brought to the casualty of our hospital with complaints of respiratory distress and endotracheal tube in situ; the child also had gangrene over the abdomen and upper and lower limbs.

How to cite this article: Anantha T, Chittaragi VB, Karthik MK, Kumar J, Kumar BS, Sai BS. A fatal, rare case of bilateral, upper, lower limbs and abdominal gangrene associated with brucellosis. J Lab Physicians 2019;11:388-90. 
A previous history of the patient revealed that the child had a fever and swelling over the abdomen for 20 days. Fever was intermittently associated with chills and rigors. Swelling over the abdomen was gradual in onset and progressed leading to abdominal distension followed by edema of limbs. This was associated with blackish discoloration of abdomen including four limbs and was noticed for 5 days. It progressed to the formation of blebs and oozing of pus. There was no history of vomiting, loose stools, convulsions, or trauma. For these chief complaints, the patient was initially taken to multiple hospitals where he was diagnosed as dengue fever (immunoglobulin $\mathrm{M}$ positive) with anterior abdominal cellulitis and was treated with multiple antibiotics. The patient was referred to Indira Gandhi Hospital, Bengaluru, in view of vascular surgery requirement, from where he was again referred to our hospital and presented with the above complaints. On admission to our hospital, his heart rate was $176 \mathrm{bpm}$, respiratory rate $76 \mathrm{cpm}$, saturation of $44 \%$ with bag and tube ventilation with blood pressure 53/35 $\mathrm{mmHg}$ and prolonged clot formation time. The child was put on ventilator with maximum ventilator parameters. Complete hemogram revealed hemoglobin of $2.9 \mathrm{mg} / \mathrm{dl}$ and total leukocyte count of 29,580 cells/cu.mm with $54 \%$ neutrophils. The patient was afebrile on admission but in due course had high-grade fever intermittently. The patient was on treatment with multiple antibiotics such as meropenem, linezolid, and clindamycin. Despite treatment with antibiotics, the patient still continued to have high-grade fever. Hence, to rule out sepsis, two blood samples were collected $1 \mathrm{~h}$ apart from two different sites under aseptic precautions and were sent to the Department of Microbiology for culture and sensitivity. The samples were loaded to BacT / ALERT 3D 60 automated blood culture system, and both the bottles were flagged positively within $48 \mathrm{~h}$ of incubation. The samples were subcultured onto blood agar, chocolate agar, and MacConkey agar for isolation of the pathogen, and the plates were incubated at $37^{\circ} \mathrm{C}$ for $24 \mathrm{~h}$. At the end of $24 \mathrm{~h}$, small, convex, smooth, translucent, nonhemolytic colonies were seen on blood agar [Figure 1], and chocolate agar and MacConkey agar showed no growth. Smear from the culture showed Gram-negative coccobacillary forms [Figure 2]. The isolate was positive for catalase, oxidase, and hydrolyzed urea rapidly within $2 \mathrm{~h}$ of incubation. The organism when subjected to VITEK 2 automated system identified the pathogen as $B$. melitensis. Considering this as critical alert and when called to inform the report to the clinician, we got to know that the patient had already expired. The patient serum sample was retrospectively subjected to standard agglutination test to know the significant titer, and the patient had a titer of 1:1280 suggestive of brucellosis [Figure 3]. According to the treating physician, the patient was infused with noradrenaline in view of shock and 1 pint of packed red blood cell, and platelet was transfused.

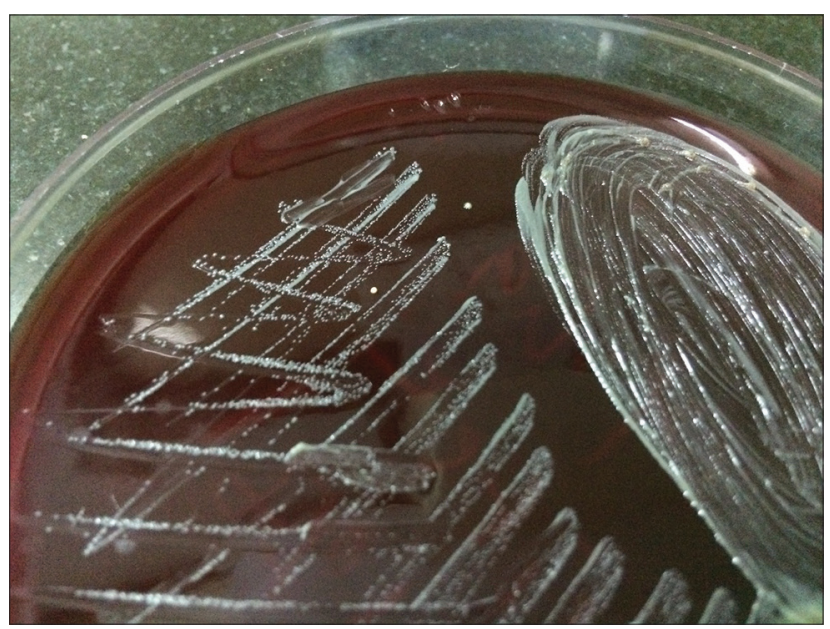

Figure 1: The growth of Brucella melitensis on blood agar

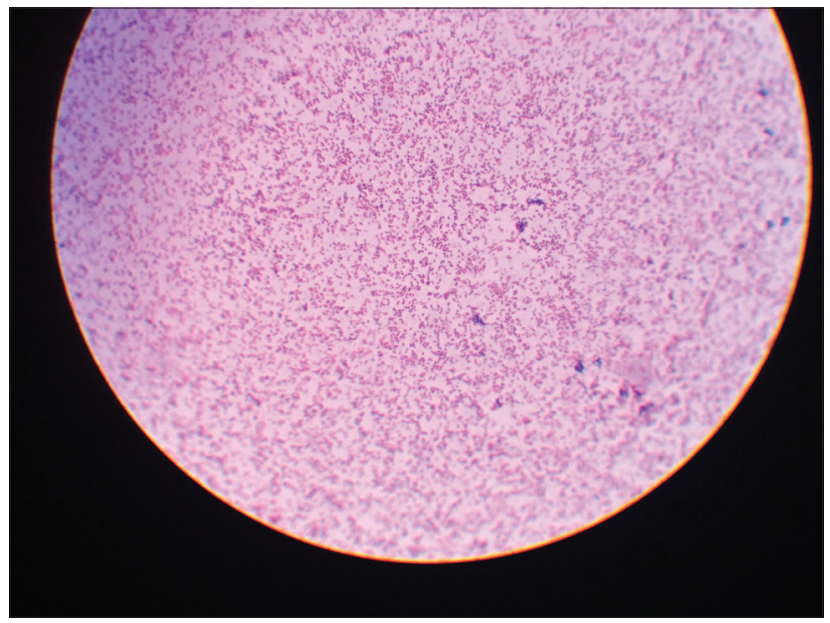

Figure 2: Gram staining picture of Brucella

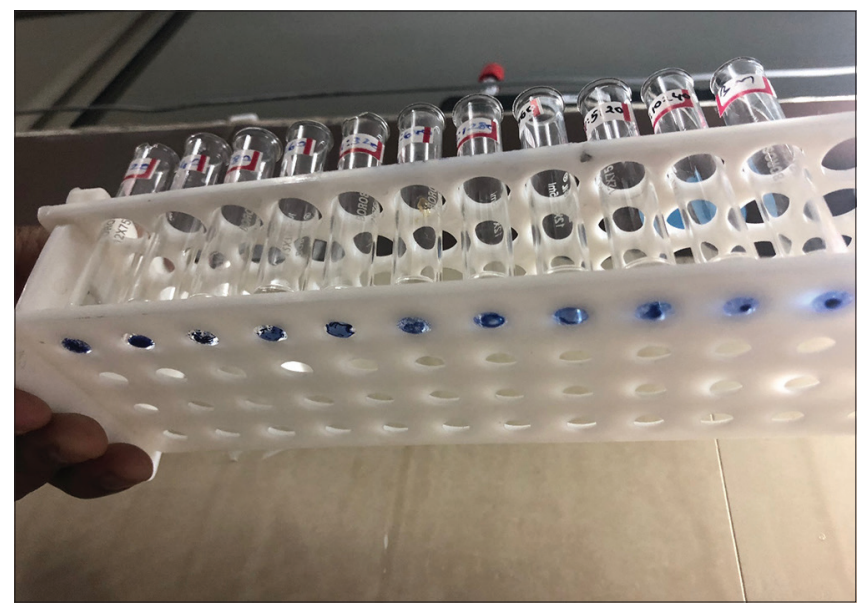

Figure 3: Standard agglutination test for brucellosis

Antibiotics were not changed due to unavailability of the reports. Gangrene was nonsalvageable which involved thrombosis of arteries and veins. The patient in due had an episode of bradycardia with desaturation, and in spite of best resuscitative measures with three doses of 
adrenaline, he could not be revived and succumbed to death.

\section{Discussion}

Brucellosis is still a serious health problem in developing countries and is commonly acquired in Mediterranean countries, the Middle East, the Arabian Peninsula, Central and South America, Asia, and Africa. This infection mimics many clinical conditions and is difficult to suspect or diagnose clinically. Hence, in any cases of pyrexia of unknown origin, brucellosis should always be considered as a differential diagnosis. ${ }^{[11]} \mathrm{Few}$ studies have well documented about transmission of brucellosis through breast milk feeding, consumption of unpasteurized milk, and occupational exposure to cattle. Hence, medical practitioners should concentrate on history of exposure and consumption of raw milk in cases with clinical suspicion of brucellosis. ${ }^{[12]}$ As our patient belonged to the pediatric age group residing in a rural background, there might be a possibility of such transmission, and we could not get the history of consumption of raw milk or contact with the cattle.

This patient presented with gangrene over all the four limbs and abdomen with continuous fever. Blood culture yielded the growth of B. melitensis. In most of the case reports, the diagnosis of brucellosis happened by unexpected isolation of Brucella from blood cultures. Although culture is a gold standard for the diagnosis of brucellosis, it can be presumptively diagnosed by demonstrating high antibody titers that are specific to Brucella antigens. Our patient had an antibody titer of 1:1280. Hence, routine serological diagnosis in patients with clinical signs and symptoms suggestive of brucellosis is essential. This also helps in detecting more number of cases. ${ }^{[6]}$ In conclusion, brucellosis still remains a serious threat and poses a severe impact on the public health concern, particularly where the disease is endemic. Hence, a high index of suspicion is really necessary when patients presenting with unexplained symptoms, especially for those who are at an occupational risk for brucellosis. Early diagnosis and prompt therapy can show an improvement in the outcome of the patients with brucellosis.

\section{Declaration of patient consent}

The authors certify that they have obtained all appropriate patient consent forms. In the form the patient(s) has/ have given his/her/their consent for his/her/their images and other clinical information to be reported in the journal. The patients understand that their names and initials will not be published and due efforts will be made to conceal their identity, but anonymity cannot be guaranteed.

\section{Financial support and sponsorship Nil.}

\section{Conflicts of interest}

There are no conflicts of interest.

\section{References}

1. Pappas G, Papadimitriou P, Akritidis N, Christou L, Tsianos EV. The new global map of human brucellosis. Lancet Infect Dis 2006;6:91-9.

2. Tuon FF, Gondolfo RB, Cerchiari N. Human-to-human transmission of brucella - A systematic review. Trop Med Int Health 2017;22:539-46.

3. Young EJ, Mandell GL, Bennett JE, Dolin R. editors. Brucella species. In: Principles and Practice of Infectious Diseases. Philadelphia, PA, USA: Churchill Livingstone; 2005.

4. Franco MP, Mulder M, Gilman RH, Smits HL. Human brucellosis. Lancet Infect Dis 2007;7:775-86.

5. Ataman C, Hatipoglu A, Yetkin G, Ertem T, Tulek N. Unusual clinical presentations of brucellosis. Scand J Infect Dis 2004;36:694-7.

6. Mantur BG, Amarnath SK, Shinde RS. Review of clinical and laboratory features of human brucellosis. Indian J Med Microbiol 2007;25:188-202.

7. Durusoy R, Karababa AO. Completeness of hepatitis, brucellosis, syphilis, measles and HIV / AIDS surveillance in Izmir, Turkey. BMC Public Health 2010;10:71.

8. Pappas G, Akritidis N, Bosilkovski M, Tsianos E. Brucellosis. N Engl J Med 2005;352:2325-36.

9. Young EJ. An overview of human brucellosis. Clin Infect Dis 1995;21:283-9.

10. Doganay GD, Doganay M. Brucella as a potential agent of bioterrorism. Recent Pat Antiinfect Drug Discov 2013;8:27-33.

11. Castaño MJ, Solera J. Chronic brucellosis and persistence of Brucella melitensis DNA. J Clin Microbiol 2009;47:2084-9.

12. al-Kharfy TM. Neonatal brucellosis and blood transfusion: Case report and review of the literature. Ann Trop Paediatr 2001;21:349-52. 